\title{
Median arcuate ligament syndrome, a rare case of chronic abdominal pain
}

\author{
Fania Puccia, ${ }^{1}$ Filippo Alessandro Montalto, ${ }^{1}$ Luigi Mirarchi, ${ }^{1}$ Antonino Terranova, ${ }^{1}$ Antonietta Serruto, ${ }^{1}$ \\ Roberto Citarrella, ${ }^{1}$ Giuseppe Lo Re, ${ }^{2}$ Maurizio Soresi ${ }^{1}$
}

${ }^{1}$ Biomedical Department of Internal Medicine and Specialties, Division of Internal Medicine, University-Hospital of Palermo; ${ }^{2}$ Section of Radiological Sciences, Di.Bi.Med., University-Hospital of Palermo, Italy

\begin{abstract}
The median arcuate ligament syndrome (MALS) is a rare disease characterized by abdominal pain caused by the external compression of the celiac artery by the median arcuate ligament. Surgical treatment is indicated, but given the non-specific symptoms, these patients are often hospitalized in the Departments of Internal Medicine where the diagnosis may be unknown. We present a case of MALS admitted to our Internal Medicine Division. An abdominal ultrasound in a woman with longstanding abdominal pain showed elevated celiac artery velocities during forced expiration. Computed tomography angiography (CTA) of the abdomen showed stenosis of the origin of the celiac artery and confirmed the diagnosis of MALS. MALS is a syndrome that has to be considered, especially in young women with abdominal pain of unclear etiology; evaluated by color Doppler ultrasound, in the presence of elevated hepatic artery velocities during forced expiration, the confirmatory test is CTA.
\end{abstract}

\section{Introduction}

The median arcuate ligament syndrome (MALS) or Dunbar Syndrome is a rare disease caused by the external compression of the celiac trunk by the MAL and characterized by abdominal pain, nausea, vomiting, weight loss. ${ }^{1}$

The MAL passes superior to the origin of the

Correspondence: Maurizio Soresi, Biomedical Department of Internal Medicine and Specialties, Division of Internal Medicine, University-Hospital of Palermo, via del Vespro 141, 90127 Palermo, Italy.

Tel.: +39.91.6552961 - Fax: +39.91.6552896.

E-mail: maurizio.soresi@unipa.it

Key words: Median arcuate ligament syndrome; celiac artery compression syndrome; abdominal pain; ultrasound; Doppler.

Contributions: FP, MS, conceived the work; FP, FAM, LM, wrote the paper; AT, AS, bibliographic research; RC, GLR, MS, discussed the critical points of case report.

Conflict of interest: the authors declare no potential conflict of interest.

Received for publication: 9 May 2018.

Accepted for publication: 30 May 2018.

This work is licensed under a Creative Commons Attribution NonCommercial 4.0 License (CC BY-NC 4.0).

(C) Copyright F. Puccia et al., 2018

Licensee PAGEPress, Italy

Italian Journal of Medicine 2018; 12:219-222

doi:10.4081/itjm.2018.1041 celiac artery; in $10 \%$ to $24 \%$ of the general population this fibrous arch is positioned lower, crossing over the proximal portion of the celiac axis, compressing it and some of these patients may have hemodynamically significant stenosis. ${ }^{1,2}$

Surgical treatment is indicated for symptomatic MALS, but given the non-specific symptoms, these patients are often hospitalized in the Departments of Internal Medicine where the diagnosis may be unknown. ${ }^{1}$

We present a case of a patient with MALS admitted to our Internal Medicine Division presenting with chronic abdominal pain and weight loss.

\section{Case Report}

A 44-year-old woman presented to the emergency room with epigastric abdominal pain.

The patient reported a 2-year history of intermittent abdominal pain associated with nausea and weight loss. The medical checks carried out at that time were normal. She had rare diarrhea and she denied alcohol or drug abuse.

The physical examination revealed mild epigastric tenderness to palpation.

Laboratory tests (complete blood counts, liver function tests, serum amylase and lipase) and esophagogastroduodenoscopy were normal.

Abdominal ultrasound ruled out cholelithiasis whereas color Doppler ultrasound (CDUS) revealed increased celiac artery velocities during forced expiration (Figure 1A and B). Suspecting MALS, a computed tomography angiography (CTA) of the 
abdomen was performed, which showed stenosis of the origin of the celiac artery caused by the median arcuate ligament with its typical hook-shaped appearance (Figures 2 and 3). These findings confirmed the diagnosis of MALS.

The patient was referred to the surgeons for a sixmonth follow-up.

\section{Discussion}

The median arcuate ligament syndrome or celiac axis compression syndrome, or Dunbar syndrome is a rare disease caused by the external compression of the celiac trunk by the median arcuate ligament. ${ }^{2}$

Given the non-specific symptoms and presentation, MALS is very difficult to be diagnosed and needs to be differentiated from other causes of abdominal pain, including biliary diseases and peptic ulcer diseases. Therefore, these patients are often hospitalized in the Departments of Internal Medicine where the diagnosis may be unknown.

This syndrome was described in 1963 by Harjola ${ }^{3}$ and in 1965 by Dunbar et al. ${ }^{4}$ and the definition is based on a combination of clinical and radiographic features. The majority of affected patients are asymptomatic due to sufficient collateral supply from superior mesenteric circulation and radiographic finding of celiac axis compression may not be significant. Severe compression occurs in approximately $1 \%$ of patients. ${ }^{3}$

The MALS's pathophysiologic mechanism is unknown but there are two main theories, mainly mesenteric ischemia and nerve dysfunction. ${ }^{5-7}$

Usually, the disease occurs in young women and is characterized by postprandial abdominal pain, weight loss, nausea, vomiting, bloating, diarrhea, reduced appetite, and the presence of extrinsic celiac compression on vascular imaging. ${ }^{2,8}$

In the past, angiography was considered the gold standard in the diagnosis of MALS. Nowadays it has been substituted by new diagnostic tools.

A color Doppler ultrasound is a good screening tool; once suspected on CDUS, the diagnosis of celiac artery compression is made by CTA and magnetic resonance angiogram (MRA). ${ }^{9}$

The mesenteric ultrasound, performed during deep expiration, shows increased blood flow velocity in celiac artery narrowing that may normalize with inspiration or with standing (erect); the main indicators for MALS are the peak systolic velocity (PSV) of $>350 \mathrm{~cm} / \mathrm{s}$ during deep expiration and a deflection angle greater than $50^{\circ} .2,10,11$

Another criterion to diagnose MALS is a PSV ratio greater than 3:1 in the celiac artery during expiration compared with the PSV in the abdominal aorta under the diaphragm. ${ }^{12}$

Sagittal view of CTA identifies a characteristic focal narrowing of the celiac artery exacerbated during the expiratory phase $;{ }^{13}$ the hooked appearance with the absence of arterial calcifications differentiate this condition from atherosclerotic disease, main causes of celiac artery narrowing. ${ }^{7,14}$

MRA can also be used in patients with allergy to iodinated contrast media and with results similar to CTA.

In our patient, CTA demonstrated focal narrowing of the celiac axis at the origin suggestive of MALS.

The treatment for symptomatic MALS patients is open surgery or laparoscopic division of the median arcuate ligament to relieve the compression ${ }^{2}$ restoring normal blood flow in the celiac axis; percutaneous endovascular treatment is an alternative technique and may be considered in selected cases. ${ }^{15}$

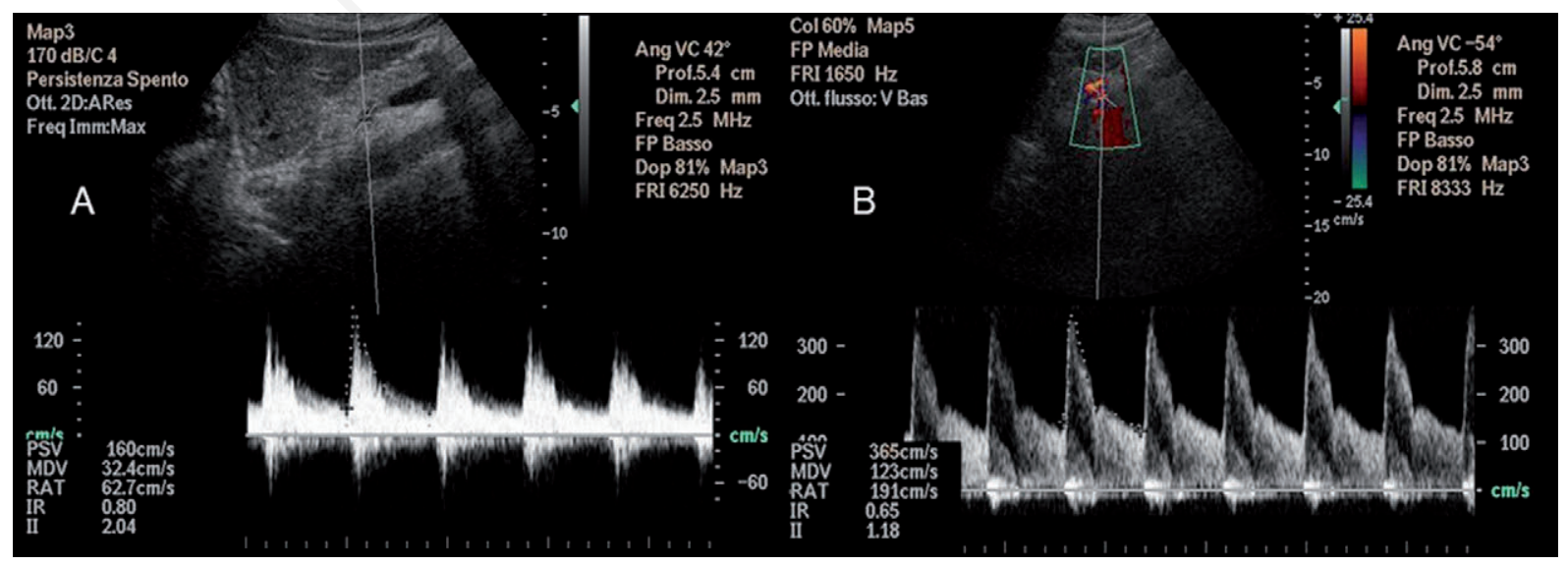

Figure 1. A) Doppler spectral analysis of the celiac artery in inspiration, flow with a peak systolic velocity of $365 \mathrm{~cm} / \mathrm{sec}$; B) color Doppler and spectral analysis of celiac artery during forced expiration, color Doppler shows flow with a peak systolic velocity of $365 \mathrm{~cm} / \mathrm{sec}$. 


\section{Conclusions}

Median arcuate ligament syndrome is a difficult diagnosis to obtain in the majority of patients due to its nonspecific symptoms. MALS is a diagnosis of exclusion and should be considered, when evaluating patients, especially young females, who present with abdominal pain of unclear etiology.

A color Doppler ultrasound to assess celiac artery velocities is a good screening tool for patients with

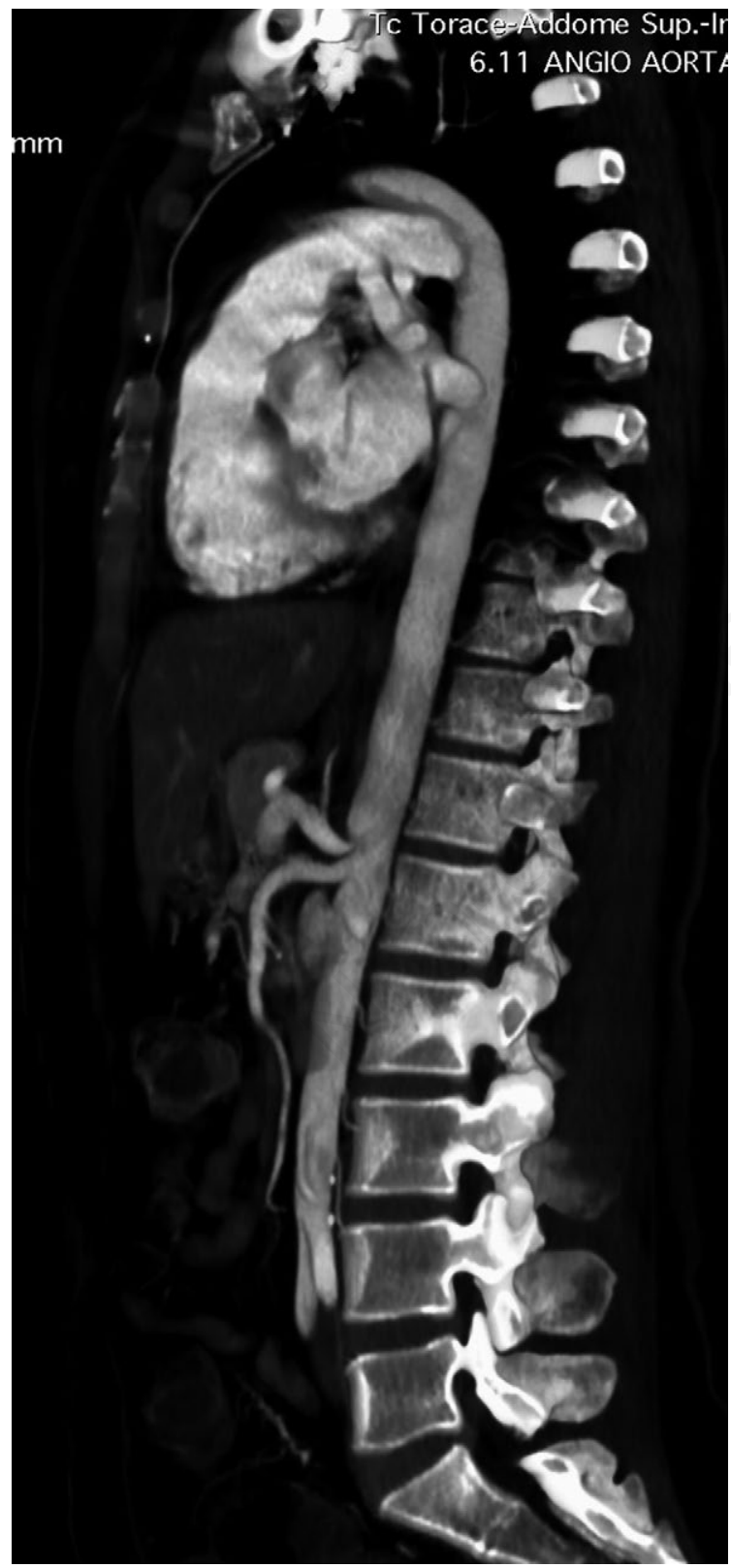

Figure 2. Reconstruction computed tomography angiography SLAB 3D in sagittal plain shows stenosis of celiac artery caused by the median arcuate ligament with its typical hook-shaped appearance. suspected median arcuate ligament syndrome. This diagnosis can be confirmed after performing a CTA.

Surgical treatment is indicated for symptomatic MALS, but given the non-specific symptoms, these patients are often hospitalized in the Departments of Internal Medicine where the diagnosis may be unknown.

\section{References}

1. Duffy AJ, Panait L, Eisenberg D, et al. Management of median arcuate ligament syndrome: a new paradigm. Ann Vasc Surg 2009;23:778-84.

2. Kim EN, Lamb K, Relles D, et al. Median arcuate ligament syndrome-review of this rare disease. JAMA Surg 2016;151:471-7.

3. Harjola PT. A rare obstruction of the coeliac artery: report of a case. Ann Chir Gynaecol Fenn 1963;52:547-50.

4.Dunbar JD, Molnar W, Beman FF, et al. Compression of the celiac trunk and abdominal angina. Am J Roentgenol Radium Ther Nucl Med 1965;95:731-44.

5. Reuter SR, Bernstein EF. The anatomic basis for respiratory variation in median arcuate ligament compression of the celiac artery. Surgery 1973;73:381-5.

6. Jimenez JC, Harlander-Locke M, Dutson EP. Open and laparoscopic treatment of median arcuate ligament syndrome. J Vasc Surg 2012;56:869-73.

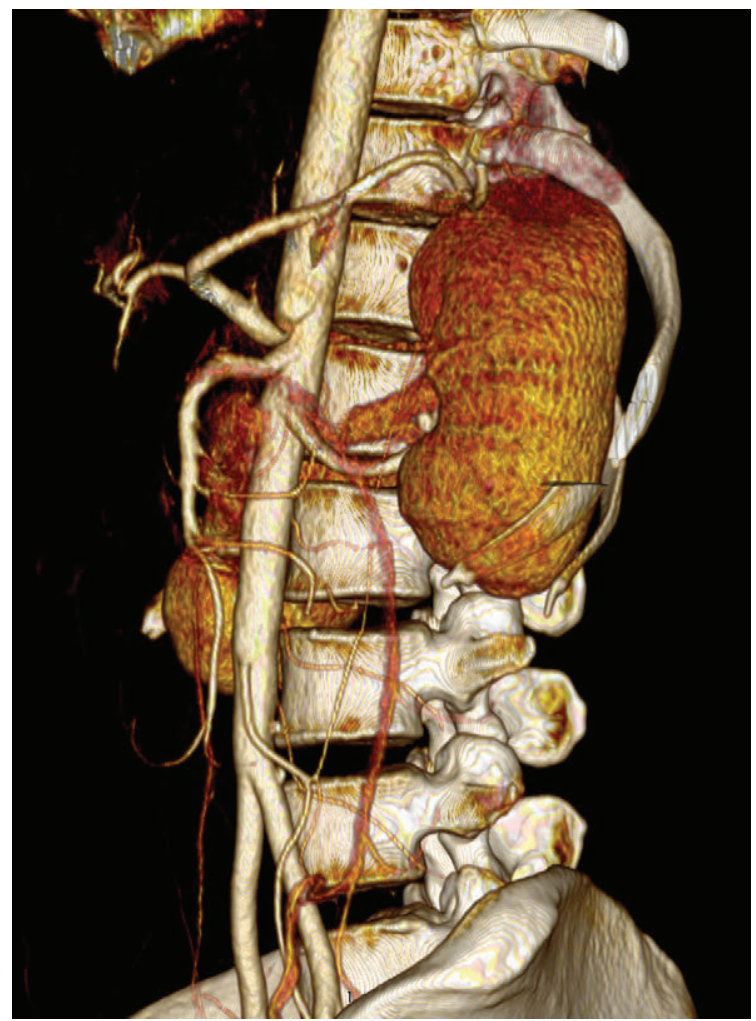

Figure 3. Reconstruction computed tomography angiography 3D volume rendering in sagittal plane: shows stenosis of celiac artery. 
7. Lainez RA, MD, Richardson WS. Median arcuate ligament syndrome: a case report. Ochsner J 2013;13:561-4.

8. Srisajjakul S, Prapaisilp P, Bangchokdee S. Imaging features of vascular compression in abdomen: fantasy, phenomenon, or true syndrome. Indian J Radiol Imaging 2017;27:216-24.

9. Kuruvilla A, Murtaza G, Cheema A, Arshad HMS. Median arcuate ligament syndrome: it is not always gastritis. J Investig Med High Impact Case Rep 2017;5:1-3.

10. Tembey RA, Bajaj AS, Wagle PK, Ansari AS. Real-time ultrasound: key factor in identifying celiac artery compression syndrome. Indian J Radiol Imaging 2015;25:202-5.

11. Gruber H, Loizides A, Peer S, Gruber I. Ultrasound of the median arcuate ligament syndrome: a new approach to diagnosis. Med Ultrason 2012;14:5-9.

12. Scholbach T. Celiac artery compression syndrome in children, adolescents, and young adults: clinical and color duplex sonographic features in a series of 59 cases. J Ultrasound Med 2006;25:299-305.

13. Ng FH, Wai OK, Wong AW, Yu SM. Median arcuate ligament syndrome. Hong Kong Med J 2016;22:184.e3-4.

14. Sunkara T, Caughey ME, Zhen KC, et al. Dunbar syndrome-a rare cause of foregut ischemia. J Clin Diagn Res 2017;11:OD13-4.

15. Hongsakul K, Rookkapan S, Sungsiri J, Tubtawee T. A severe case of median arcuate ligament syndrome with successful angioplasty and stenting. Case Rep Vas Med 2012;2012:129870. 